\title{
Long-term macrolide treatment for chronic respiratory disease
}

\author{
Paolo Spagnolo ${ }^{1}$ Leonardo M. Fabbri ${ }^{1}$ and Andrew Bush ${ }^{2}$
}

\begin{abstract}
Affiliations:
${ }^{1}$ Section of Respiratory Diseases, Dept of Medical and Surgical Sciences for Children and Adults, University of Modena and Reggio Emilia, Policlinico di Modena, Modena, Italy.

${ }^{2}$ Imperial College and Royal Brompton Harefield NHS Foundation Trust, London, UK.

Correspondence:

P. Spagnolo, Section of Respiratory Diseases, Dept of Medical and Surgical Sciences for Children and Adults, University of Modena and Reggio Emilia, Policlinico di Modena, Via del Pozzo 71, Modena 41100, Italy.

E-mail: paolo.spagnolodunimore.it
\end{abstract}

ABSTRACT Long-term macrolide treatment was first shown to alter the natural history of diffuse panbronchiolitis (DPB) in the late 1980s. Since then, macrolides have been demonstrated to exert antiinflammatory and immunomodulatory activity in addition to being antimicrobial. Indeed, their spectrum of action extends to the regulation of leukocyte function and production of inflammatory mediators, control of mucus hypersecretion, resolution of inflammation and modulation of host defence mechanisms. As such, the potential benefit of macrolide antibiotics has been evaluated in a variety of chronic respiratory diseases. The best studied condition is cystic fibrosis, of which there have been six randomised controlled trials showing evidence of benefit. However, most of the studies were limited by small numbers of patients and short follow-up. More recently, landmark studies have demonstrated the efficacy of azithromycin in reducing the risk of acute exacerbations in patients with chronic obstructive pulmonary disease, but the optimal duration and dosing of macrolide treatment remain uncertain.

With the exception of patients with DPB and cystic fibrosis, until clear evidence of efficacy is available, the long-term use of macrolides should be limited to highly selected patients after careful evaluation of benefit and harm, or in the context of randomised controlled clinical trials.

@ERSpublications

Several studies evaluating macrolide antibiotics for the treatment of chronic respiratory diseases are underway http://ow.ly/kFU2F

Received: Aug 292012 | Accepted after revision: Nov 072012 | First published online: Nov 222012

Support statement: A. Bush was supported by the National Institute for Health Research Respiratory Disease Biomedical Research Unit at the Royal Brompton and Harefield NHS Foundation Trust and Imperial College London (London, UK).

Conflict of interest: Disclosures can be found alongside the online version of this article at www.erj.ersjournals.com 


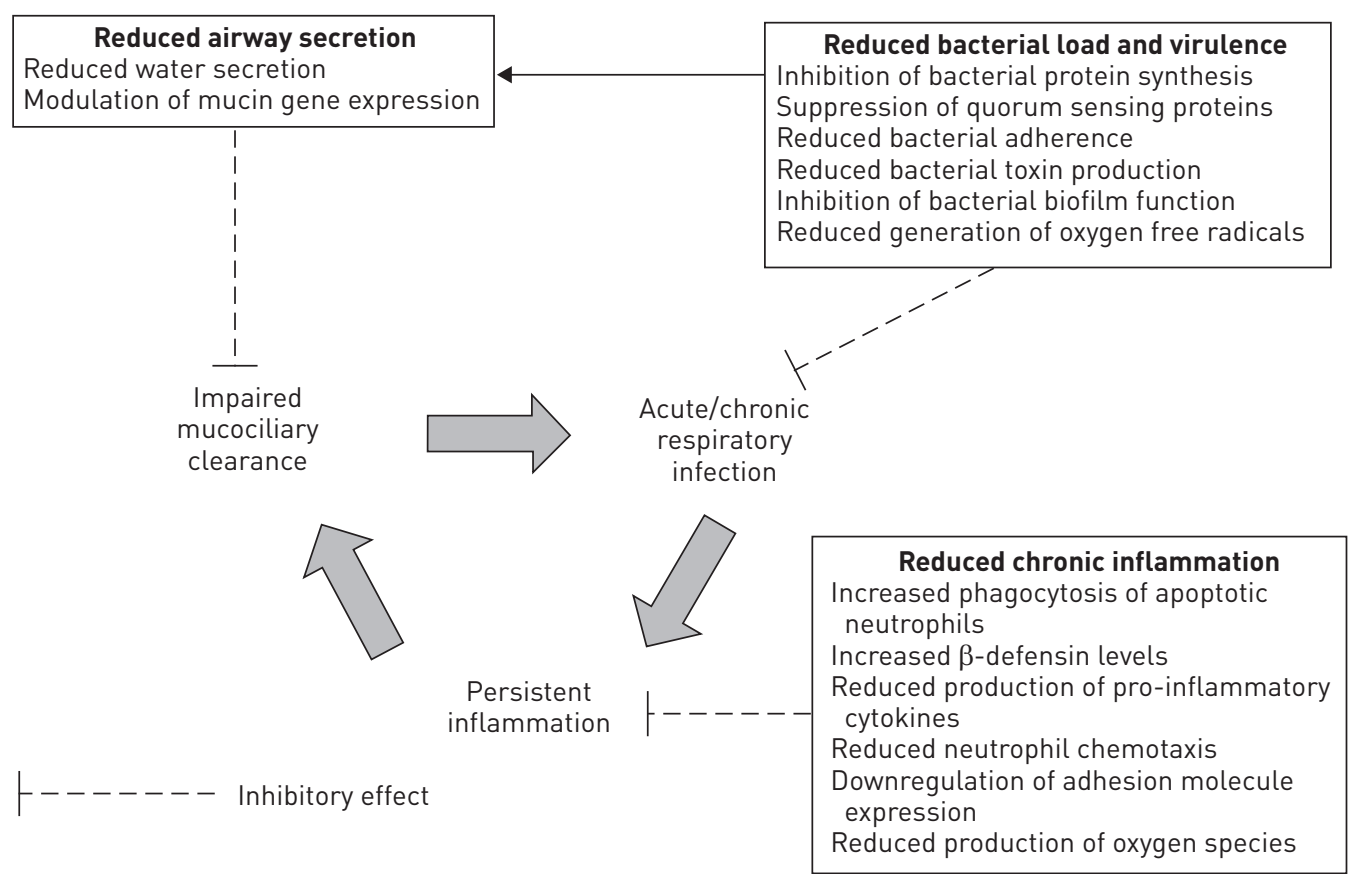

FIGURE 1 Potential beneficial effects of macrolides in chronic respiratory diseases. Macrolides inhibit bacterial protein synthesis, reduce bacterial adherence and bacterial toxin production, inhibit biofilm function and reduce the generation of oxygen free radicals, which result in reduced bacterial load and virulence; macrolides can also modulate mucin gene expression and mucin protein production, and suppress quorum sensing proteins with reduced airway secretion and improved mucociliary clearance. Finally, macrolide antibiotics exert several anti-inflammatory and immunomodulatory activities, including: decreased neutrophil chemotaxis and survival; downregulation of adhesion molecule expression; increased alveolar macrophage phagocytosis of apoptotic cells; downregulation of adhesion molecule expression; inhibition of transcription factors leading to decreased pro-inflammatory cytokine production; increased $\beta$-defensin levels; reduced oxygen species production; reduction in T-cell number and migration; and modulation of dendritic cell function, thus attenuating chronic inflammation.

\section{Introduction}

Macrolide antibiotics, commonly referred to as "macrolides", belong to a family of compounds chemically characterised by the presence of a macrocyclic lactone ring of $\geqslant 12$ elements [1]. Given their favourable bioavailability via the oral route, excellent tissue penetration and broad efficacy against many lung pathogens (most Gram-positive and some Gram-negative bacteria, mycobacteria, Chlamydia, Mycoplasma and Legionella species), macrolides are widely used as first-line agents in the therapy of respiratory infections [2], although there is a concern that long-term administration of macrolides can promote antimicrobial resistance [3]. In recent years, there has been an increasing interest in the potential immunomodulatory properties of macrolides following the observation of the effectiveness of erythromycin in diffuse panbronchiolitis (DPB), an idiopathic disease found almost exclusively in the Far East and characterised by chronic recurrent bronchiolitis and peribronchiolitis with inflammatory infiltration of the small airways [4]. The immunomodulatory effects of macrolides, which are only seen with 14(erythromycin, clarithromycin and roxithromycin) and 15-members (azithromycin) [5], may take several weeks to manifest, and include reduced airway mucus secretion and viscosity [6] and decreased airway neutrophil accumulation through a reduction in pro-inflammatory cytokines expression and adhesion molecule production [7-9] (fig. 1).

Collectively, these observations provided the rationale for a number of studies performed over the last decade to assess the efficacy of macrolides in chronic respiratory diseases other than DPB, such as cystic fibrosis (CF), asthma, chronic obstructive pulmonary disease (COPD), non-CF bronchiectasis, and bronchiolitis obliterans syndrome (BOS) [10]. The purpose of this review is to summarise and discuss the potential for macrolide therapy in chronic respiratory diseases in the light of the data provided by recently concluded large clinical trials.

\section{Search strategy and selection criteria}

We conducted a literature search using the Pubmed/MEDLINE and EMBASE databases. We used the terms "macrolide" OR “erythromycin” OR “clarithromycin” OR “azithromycin” OR "roxithromycin” OR 
FIGURE 2 Diffuse panbronchiolitis. High resolution computed tomography image through the right middle and lower lobes, showing a profusion of small nodules and branching structures (treein-bud pattern) with accompanying cylindrical bronchiectasis. Personal communication: N. Sverzellati, University of Parma, Parma, Italy.

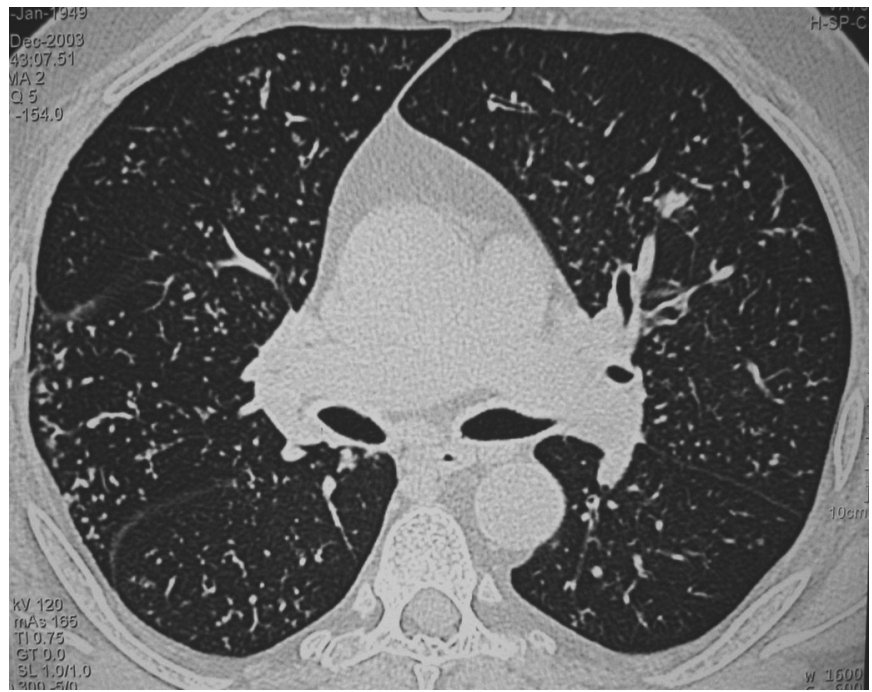

"troleandomycin" OR "telithromycin" in combination with the terms "asthma", "bronchiectasis", "cystic fibrosis", "diffuse panbronchiolitis", "bronchiolitis obliterans", "chronic rhinosinusitis", "chronic obstructive pulmonary disease", "COPD”, “organising pneumonia”, "acute lung injury" and "respiratory viral infections". Our review includes only items published in the past 3 years and is not limited to doubleblind placebo-controlled studies.

\section{Diffuse panbronchiolitis}

DPB is a progressive inflammatory airway disease reported almost exclusively in East Asians and characterised by chronic airway infection, often complicated by Pseudomonas aeruginosa infection, chronic productive cough, dyspnoea, airflow limitation and chronic sinusitis [11, 12]. The term "diffuse" refers to the distribution of the lesions throughout both lungs, while "pan-" refers to the inflammatory involvement of all layers of the respiratory bronchioles. Radiographic findings include reticulo-nodular infiltrates, while high-resolution computed tomography (HRCT) scans show the characteristic centrolobular nodules with a "tree-in-bud" appearance (fig. 2). Bronchoalveolar lavage fluid (BALF) obtained from DPB patients reveals high levels of neutrophils and lymphocytes, as well as of interleukin (IL)-8 and other pro-inflammatory cytokines and chemokines $[13,14]$, suggesting a chronic inflammatory process further exacerbated by the presence of pathogens.

KUDOH et al. [4] were the first to demonstrate in a large retrospective study that low-dose erythromycin not only ameliorated the signs and symptoms of DPB but also improved survival. These unexpected results were attributed to a previously unknown anti-inflammatory effect of erythromycin rather than to its antimicrobial properties. In fact, erythromycin $600 \mathrm{mg}$ daily for a month has been shown to reduce the number of neutrophils and the concentration of IL- 8 in BALF of DPB patients regardless of P. aeruginosa infection [15]. In addition, the maximal serum and sputum levels of erythromycin have been reported to be below the minimum inhibitory concentration of the relevant pathogens (Haemophilus influenzae and $P$. aeruginosa), thus confirming that it is unlikely that the beneficial effect of erythromycin in DPB purely results from its antibacterial activity [16].

Other macrolide antibiotics that have proved effective in DPB include roxithromycin, clarithromycin and azithromycin [17-19]. The treatment of DPB is the most striking example of the benefits of using macrolides to treat chronic respiratory diseases, with the 10-year survival rate increasing from $12-50 \%$ (depending on whether patients were infected with $P$. aeruginosa) to $>90 \%$ since the introduction of macrolide therapy $[4,20]$. So successful has this treatment been that it is impossible nowadays to conduct a randomised controlled trial (RCT), since no patient will agree to be randomised to placebo. Nevertheless, the authors of a recent systematic review evaluating the effects of macrolides in DPB identified only one small randomised controlled trial of poor methodological quality [21] and concluded that the use of macrolides in DPB is based on non-RCTs or retrospective studies [22].

\section{Cystic fibrosis}

$\mathrm{CF}$, the most common life-shortening inherited disease in white populations ( 1 in 2500 newborns in white people), is caused by mutations in the cystic fibrosis transmembrane conductance regulator (CFTR) gene [23]. 


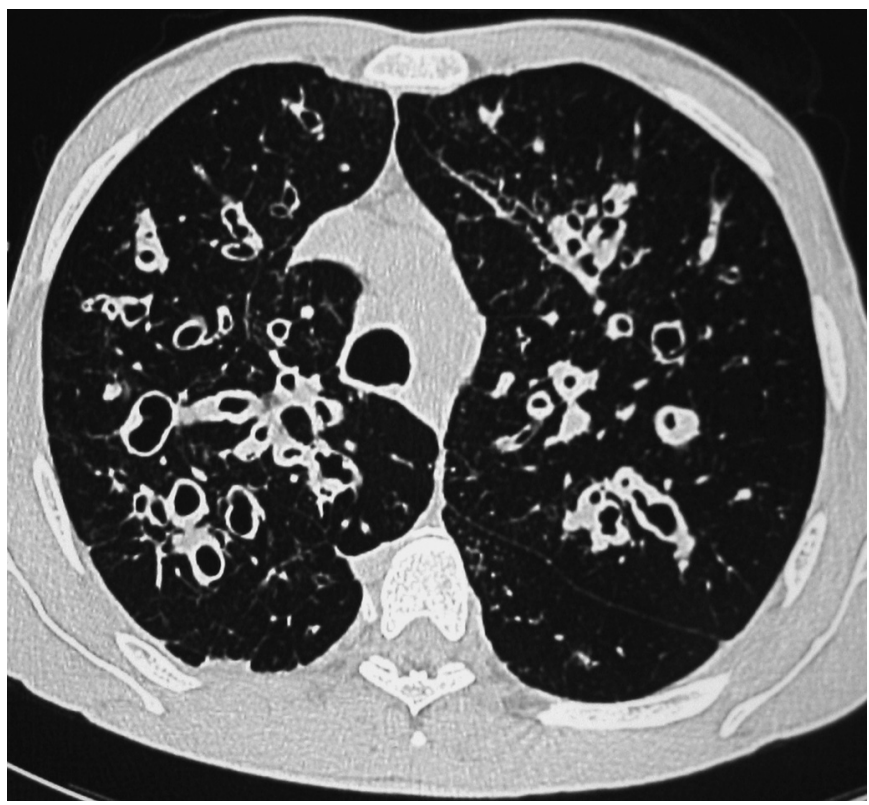

FIGURE 3 Cystic fibrosis. Upper lobe predominant bronchiectasis with large mucus plugs in a 14-year-old patient.

Alterations in epithelial cell ion transport resulting from defective CFTRs lead to increased sputum viscosity, stasis of secretions, impaired mucociliary clearance, recurrent respiratory infections and chronic progressive bronchiectasis, in a vicious circle (fig. 3) [24]. In CF, abnormal CFTR function affects a number of organs, but the involvement of the airways has the most dramatic impact on quality of life and survival [25]. Characteristic organisms associated with airway infection in patients with CF are, most notably, Staphylococcus aureus in the early course of the disease and $P$. aeruginosa at a later stage [26]. However, there is increasing appreciation that the lower airways of CF patients harbour communities of bacteria far more complex than previously thought and which include, amongst others, Stenotrophomonas maltophilia, isolates of the species Burkholderia cepacia, nontuberculous mycobacteria, fungi and viruses [27-29].

The airway inflammatory response in CF is characterised by neutrophilic infiltration and pro-inflammatory cytokine production. This has been demonstrated even in the absence of bacterial infection, suggesting that airway inflammation may precede bacterial infection [30]; however, it remains controversial whether the CF airway is intrinsically pro-inflammatory in the absence of infection, or whether there is solely an augmented inflammatory response in the presence of infection. The possibility that macrolides might modify the neutrophilic inflammatory response in CF was first investigated in the early 2000s. Since then, a number of clinical trials have confirmed their beneficial effects both in adults and children [31-37]. Recently, in a multicentre, randomised, double-blind, placebo-controlled trial, 260 CF patients, not infected with P. aeruginosa, were randomised in a 1:1 ratio to azithromycin $250 \mathrm{mg}$ or $500 \mathrm{mg}$ (based on body weight) 3 days per week, or placebo, for a period of 24 weeks. Azithromycin treatment did not improve pulmonary function, suggesting that the beneficial effect of azithromycin in CF is mainly due to its activity against $P$. aeruginosa [36]. However, it should be noted that the vast majority of the recruited patients had very good lung function (mean forced expiratory volume in $1 \mathrm{~s}$ (FEV1) nearly $100 \%$ predicted in both groups), thus reducing the chance of improving lung function with any intervention. This group also reported the effects of azithromycin on airway inflammation. They measured serum absolute neutrophil count, highsensitivity C-reactive protein (HsCRP), myeloperoxidase, serum amyloid A (SAA) and calprotectin, and demonstrated a significant decrease in all parameters from baseline to day 28 in the azithromycin group. The effects on neutrophil count, SAA and HsCRP were still present at the end of the study at day 168. There were some correlations between individual inflammatory parameters and weight and spirometric changes, but, in our view, these data did not show any consistent picture that would suggest that these were really important changes [38]. Azithromycin has no direct bactericidal effect on P. aeruginosa, but may reduce the organism virulence by altering biofilm formation, decreasing bacterial adherence to epithelial cells, inhibiting bacterial motility or acting synergistically with other antibiotics [39]. However, further analysis of this same dataset showed that in patients not infected with $P$. aeruginosa, azithromycin significantly reduced neutrophil counts and serum inflammatory markers within 28 days of initiating treatment [38]. As such, the mechanism/s by which macrolides exert their beneficial effect in CF (antimicrobial activity, immunomodulation or both) remains to be determined. However, a recent Cochrane systematic review and meta-analysis of data from high-quality clinical trials provides compelling evidence that long-term 
macrolide therapy improves lung function, reduces the risk of infective exacerbations, decreases the requirement for additional antibiotics and improves nutritional measures in patients with $\mathrm{CF}$, although it is unclear whether the clinical benefits may be maintained in the longer term, beyond 12 months [40]. Treatment with azithromycin was associated with reduced isolation of $S$. aureus on respiratory culture, and no change in the prevalence of atypical mycobacteria, but also with a significant increase in macrolide bacterial resistance. Adverse events were uncommon and not obviously associated with azithromycin, although a once-weekly high-dose regimen was associated with more frequent gastrointestinal adverse events. Macrolide antibiotics, other than azithromycin, have either been evaluated in underpowered studies (mostly reported in abstract form) or have been shown to be ineffective in patients with CF. RoBINSON et al. [41] performed a 12-month, double-blind crossover trial in which 63 patients were randomly assigned to receive $500 \mathrm{mg}$ oral slow-release clarithromycin or placebo once a day. 52 patients completed the study. No significant difference in either the primary efficacy end-point (change in lung function during the study period) or any secondary end-points, including, amongst others, number of pulmonary exacerbations and sputum inflammatory cytokine content, were observed during the study period between the treatment and placebo arms.

In summary, $\mathrm{CF}$ is the disease outside $\mathrm{DPB}$ for which there is most evidence for sustained benefit from macrolide therapy.

\section{Non-CF bronchiectasis}

The effectiveness of long-term macrolide therapy in DPB and CF has provided the rationale for using these agents in patients with bronchiectasis not caused by CF (commonly referred to as idiopathic bronchiectasis, although often the cause of the bronchiectasis is known, such as ciliary dyskinesia, allergic bronchopulmonary aspergillosis or post-infection) (fig. 4). Regardless of the cause, bronchiectasis involves a vicious cycle of infection leading to airway inflammation and lung damage [42]. The airway inflammatory response is characterised by an intense cellular infiltrate with mononuclear cells $\left(\mathrm{CD} 4^{+} \mathrm{T}\right.$-lymphocytes and macrophages), a prominent neutrophilia and increased IL-8 expression [43]. According to the vicious cycle hypothesis, if infection is the primary driver of airway inflammation, bacterial clearance through the use of short- or long-term antibiotic therapy would be expected to reduce airway inflammation, allow airway healing and modify the long-term course of the disease. Until very recently, the role of macrolide therapy in non-CF bronchiectasis had only been investigated in studies that were small, of short duration and did not assess clinical relevant outcomes, such as pulmonary exacerbations and quality of life [44-47]. In a recently published, randomised, double-blind, placebo-controlled trial, 141 patients (aged $\geqslant 18$ years) with a diagnosis of bronchiectasis as defined by HRCT and at least one pulmonary exacerbation requiring antibiotic treatment in the previous year, were assigned to receive azithromycin $500 \mathrm{mg}$ three times per week or placebo for 6 months in a 1:1 ratio [48]. The rate of event-based exacerbations, one of the primary end-points, was 0.59 per patient in the azithromycin group and 1.57 per patient in the placebo group during the 6-month treatment period $(\mathrm{p}<0.0001)$. Conversely, pre-bronchodilator FEV1 and St George's Respiratory Questionnaire total score, both co-primary end-points, did not differ significantly between the azithromycin and placebo groups.

FIGURE 4 Bronchiectasis in a young patient with asthma complicated by allergic bronchopulmonary aspergillosis. Mucoid impaction and small nodular branching opacities were also present in the right lung.

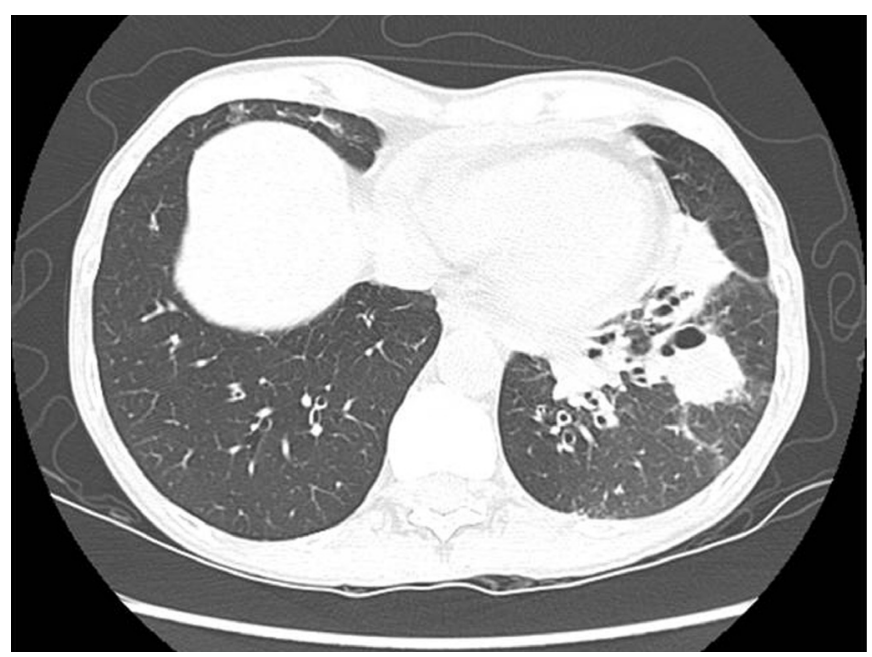


Given the small number of studies, most of which were performed on a small number of patients, the role of long-term macrolide therapy in non-CF bronchiectasis remains unclear, thus questioning the hypothesis that antibiotics can "break the cycle", and highlighting the difficulty in extrapolating from CF studies to non-CF bronchiectasis. As such, chronic macrolide therapy for this condition cannot be routinely recommended. In contrast, azithromycin may represent a therapeutic option in selected patients, i.e. those with a history of frequent exacerbations.

\section{Asthma}

Asthma is an airway disease characterised by chronic inflammation, bronchial hyperresponsiveness and airflow limitation, which manifests clinically with recurrent cough, wheezing, chest tightness, dyspnoea and mucus production [49]. The mechanisms responsible for the maintenance of the inflammatory response, which is characterised by increased numbers of activated lymphocytes, eosinophils and variably increased mast cells, are only partially known, but a growing body of evidence suggests that chronic or subacute infection with atypical bacteria, such as Mycoplasma pneumoniae and Chlamidophila pneumoniae may be an important contributor to both disease pathogenesis and severity in some patients [50]. Indeed, the very nature of infection caused by these agents, a chronic intracellular inflammatory process in the case of C. pneumoniae, and persistent epithelial damage in the case of $M$. pneumoniae, makes them ideal candidates to produce chronic symptoms and poor asthma control.

The possibility of chronic infection with organisms that are sensitive to macrolides provides a strong rationale for their use in asthma, although macrolides may also function as steroid-sparing or antiinflammatory agents [51, 52]. Early studies have reported conflicting results [53-57]. Recently, SuTHERLAND et al. [58] evaluated the effect of 16 weeks of either clarithromycin or placebo, added to fluticasone, on asthma control in individuals with or without lower airway PCR evidence of M. pneumoniae or C. pneumoniae. Due to the small number of patients with PCR positivity (12 out of 92), the two groups were combined for the analysis. The addition of clarithromycin did not improve asthma control or lung function for the entire group, suggesting that the routine addition of macrolide therapy to uncontrolled asthmatics on low-dose inhaled corticosteroids offers little additional benefit.

Interpretation of the studies as a whole is difficult owing to the heterogeneity of the study populations, the small number of patients and the relatively short treatment duration in most studies ( $\leqslant 12$ weeks). This was also reflected in a Cochrane review [59], which concluded that there is insufficient evidence to support or refute the use of macrolides in the treatment of asthma.

The evidence for a benefit from the use of macrolides in paediatrics is even more scant. Of note, a study which compared azithromycin with montelukast in children with asthma still symptomatic despite inhaled corticosteroids and long-acting $\beta_{2}$-agonists ended in futility [60]. This was because most children who were assessed either did not have asthma or were not taking their treatment, underscoring the need to get basic management steps right before escalating medical therapy.

At present, there is little evidence to justify the routine long-term use of macrolides in asthma. However, a subgroup of patients, i.e. those with evidence of atypical bacterial infection in the airways, may benefit from macrolide treatment.

\section{COPD}

COPD is the fourth leading cause of death worldwide [61]. Contributing substantially to the morbidity and mortality of patients with COPD are episodes of increased respiratory and systemic symptoms, commonly caused by airway bacterial and viral infections, referred to as acute exacerbations (AECOPD). Each year, on average, up to one-third of patients with COPD experience one or more exacerbations [62], which represent a major cause of primary care visits, hospital admission, impaired health status and mortality [63, 64]. In addition, acute exacerbations account for a substantial percentage of the cost of treating COPD [65]. As such, reduction of these events has become a major goal in the development of new therapeutic strategies for COPD. Inhaled corticosteroids, long-acting $\beta_{2}$-agonists, long-acting muscarinic antagonists, as well as roflumilast, an oral, selective phosphodiesterase type-4 inhibitor, have all been shown to reduce the frequency of acute exacerbations of COPD [66-70], but these strategies appear to reduce AECOPD at best by $40 \%[71]$.

It has been speculated that alternative approaches that directly address the infectious and inflammatory aspects of AECOPD (i.e. the use of antibiotics prophylactically) might have a significant additional benefit in terms of exacerbation reduction [72]. A number of studies have evaluated whether long-term macrolide treatment decreases the risk of AECOPD, with conflicting results [73-79]. More recently, in a prospective, parallel-group study, 1142 patients with COPD at increased risk of exacerbation were randomly assigned in a $1: 1$ ratio to receive azithromycin $(n=570)$ at a dose of $250 \mathrm{mg}$ daily or placebo $(n=572)$ for 1 year in 
addition to their usual care [80]. The median time to the first acute exacerbation of COPD (the primary outcome) was 266 days in the azithromycin group, compared with 174 days in the placebo group $(\mathrm{p}<0.001)$. In addition, azithromycin treatment decreased the frequency of AECOPD $(\mathrm{p}=0.01)$ and the incidence of colonisation with selected respiratory pathogens and improved quality of life, but was associated with an increased incidence of colonisation with macrolide-resistant organisms and decreased hearing.

Chronic bacterial colonisation of the lower airways perpetuates inflammation and contributes to the progression of COPD [81]. In turn, inflammation makes the lungs of COPD patients more susceptible to infections, both acute and chronic. Although their precise mechanisms of action (anti-infective, antiinflammatory or both) are incompletely understood, macrolides have the potential to break the vicious circle of infection-inflammation and strengthen lung defences in COPD patients at increased risk of exacerbations. Until clearer evidence of efficacy and safety is available, routine use of azithromycin prophylaxis for preventing acute exacerbations is not recommended due to an unfavourable balance between benefits and side-effects [82]. However, it may be considered for individual severe COPD patients with frequent exacerbations despite best quality, guidelines-based treatment, in those who are not at high risk of cardiovascular complications, with close monitoring of hearing, and after careful review of all medications in order to avoid any potential adverse interactions [80].

\section{Post-transplant bronchiolitis obliterans syndrome}

BOS, one of the most common and severe noninfectious pulmonary complications of chronic rejection (lung or bone marrow), is characterised by an inflammatory response of terminal and respiratory bronchioles, ultimately leading to scarring and total occlusion of the conducting airways [83] (fig. 5). Given the substantial morbidity and mortality associated with BOS, combined with the limited effectiveness of traditional antirejection therapy, and based on similarities with other bronchiolar disorders, there has been recent interest in the potential role of macrolides in the management of post-transplant BOS.

The efficacy of azithromycin (250 mg three times weekly for an mean of 16 months) was evaluated in a large observational study of lung transplant patients; 24 (30\%) out of 81 showed improvement in FEV1 after 6 months, but 22 out of the 24 responders improved after only 3 months of therapy [84]. Of note, responders at 6 months had higher pre-treatment BAL neutrophil count, with a cut-off value of $<20 \%$ having a negative predictive value of 0.91 for treatment response. Similar results had been reported by VERLEDEN et al. [85]. These studies revealed a dichotomy in the clinical spectrum of BOS, with neutrophilic (partially) reversible allograft dysfunction being macrolide responsive, while fibroproliferative BOS was not [86]. Azithromycin treatment has also been associated with increased survival. In a large retrospective cohort study of lung transplant recipients $(n=178)$, this beneficial effect was more pronounced when treatment was initiated during BOS stage 1 [87].

The potential role of azithromycin in halting progression in patients with BOS is intriguing. However, longterm randomised placebo-controlled clinical trials are required before routine use of this therapy.

\section{Chronic rhinosinusitis}

Chronic rhinosinusitis (CRS) is the second most prevalent self-reported chronic condition in the USA, affecting $\sim 15 \%$ of the population [88]. CRS, which is characterised by hyperplasia, hypertrophy and

FIGURE 5 Constrictive bronchiolitis in a patient who has undergone bone marrow transplantation. There is a mosaic attenuation pattern and the calibre of the pulmonary vessels within the areas of decreased attenuation is reduced. Thickwalled and dilated subsegmental bronchi are also present.

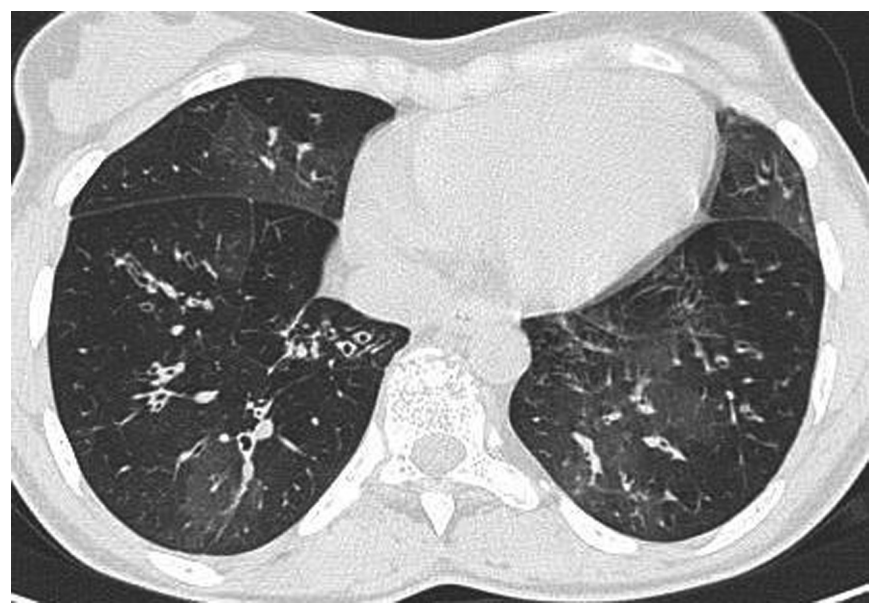


hypersecretion of the nasal and paranasal sinus mucosa, is diagnosed when symptoms of acute rhinosinusitis persist for $>12$ weeks. Long-term macrolide therapy has been shown to improve symptoms, shrink the size of nasal polyps, and decrease the levels of pro-inflammatory cytokines in patients with CRS, although mainly in small, open-label studies [89-92]. As with other diseases, the mechanisms behind this beneficial effect are unclear and may relate to the ability of macrolides to inhibit the local host immune response rather than via their antimicrobial properties [93, 94].

However, a recent study including 60 patients with recalcitrant CRS (with and without nasal polyps) unresponsive to optimal medical or surgical treatment, reported no significant differences in symptom scores or objective measures after a 3-month course of azithromycin compared with placebo [95], highlighting the need for matching patient characteristics, drug-administered and outcome measures before comparing the results of different studies.

Despite the potential interest of this therapeutic approach, current guidelines do not recommend macrolide treatment as standard therapy in CRS [96]. In addition, because of the concern for an increasing incidence of macrolide-resistant bacterial strains, repeated nasal cultures should be performed in patients on longterm macrolide therapy.

\section{Other conditions}

Recent experimental observations, mostly based on animal models, suggest that macrolide therapy may be beneficial in a number of other chronic respiratory diseases, particularly diffuse parenchymal lung disease and lung fibrosis [97]. In fact, macrolides are thought to take part in the reparative response of alveolar epithelium to injury, by acting on several components of the regenerative process, and in lipid metabolism and alveolar surfactant homeostasis [98]. However, at present, clinical evidence of efficacy in these conditions is limited to case reports or small case series.

\section{Organising pneumonia}

Organising pneumonia (OP) is an inflammatory disorder affecting the distal airways and alveoli, characterised histopathologically by intra-alveolar buds of granulation tissue, consisting of intermixed myofibroblasts and connective tissue. The disease can be either idiopathic (cryptogenic organising pneumonia (COP)) or associated with a number of entities, such as infections, drug toxicity, connective tissue disease, vasculitis, haematologic malignancies, organ transplantation, radiation therapy, eosinophilic pneumonia and many others [99]. STOVER and MANGINO [100] reported six patients (three with COP and three with OP secondary to radiation therapy) who responded to clarithromycin. The authors suggest considering long-term macrolide therapy in patients with minimal symptoms and/or minimal physiological impairment, as adjuvant therapy in patients receiving steroids or in those who cannot tolerate steroids. The beneficial effect of macrolides in OP is thought to be due to their inhibitory effect on IL- 8 release and neutrophil accumulation in the peripheral airways [101]. However, which patients are likely to respond to macrolide therapy and the appropriate dose and duration of treatment are unknown. As such, the decision to use macrolides in OP should be made on a case-by-case basis. At present, corticosteroids represent the treatment of choice for COP.

\section{Acute lung injury}

Acute lung injury (ALI) is a syndrome of acute inflammatory pulmonary oedema poorly responsive to pharmacological treatment and with a mortality rate of 30-40\% [102]. Recently, in a secondary analysis of a large multicentre clinical trial dataset, WALKEY and WIENER [103] evaluated the association between macrolide use and mortality in patients with ALI. Among patients who received a macrolide antibiotic (47 (20\%) out of 235$)$, erythromycin was the most common (57\%), followed by azithromycin (40\%) and clarithromycin (3\%). The median duration of macrolide therapy was 4 days. $11(23 \%)$ out of the 47 patients who received macrolides died, compared with $67(36 \%)$ out of the 188 who received a nonmacrolide antibiotic (either fluoroquinolone or cephalosporin; $\mathrm{p}=0.11$ ). In addition, macrolide use was associated with lower 180 -day mortality $(\mathrm{p}=0.028)$ and shorter time to successful discontinuation of mechanical ventilation $(\mathrm{p}=0.009)$. Interestingly, subjects administered macrolides were more likely to have pneumonia as a risk factor for ALI. In contrast, fluoroquinolone and cephalosporin use was not associated with improved outcomes. These findings suggest that macrolide antibiotics are promising as a potential therapy early in the course of ALI.

\section{Respiratory viral infections}

Bronchiolitis is a serious, potentially life-threatening respiratory illness that often affects young babies. It frequently occurs in the first year of life and represents the most common cause of hospital admission in babies aged $<6$ months [104]. Babies usually present with runny nose, cough, shortness of breath and signs 
of respiratory distress. Respiratory syncytial virus (RSV) is the most common pathogen identified, but other viruses, such as human meta-pneumovirus, influenza, parainfluenza, adenovirus and rhinovirus, have also been implicated [105]. The disease is poorly responsive to treatment, including antiviral drugs. Since viruses are potent inducers of cytokine and chemokine production and release [106], the potential benefit of macrolide anti-inflammatory and immunomodulatory activities has also been evaluated in respiratory viral infections, although in small studies and with conflicting results.

In a double-blind, randomised, placebo-controlled trial, TAHAN et al [107] evaluated the efficacy of clarithromycin given daily for 3 weeks at a dose of $15 \mathrm{mg} \cdot \mathrm{kg}^{-1}$ in infants aged $<7$ months hospitalised for RSV bronchiolitis. Nine subjects were excluded from analysis due to corticosteroid use, leaving 12 in the clarithromycin group and nine in the placebo group. Clarithromycin treatment was associated with a statistically significant reduction in the length of hospital stay, duration of oxygen use, need for $\beta_{2}$-agonists and readmission to the hospital within 6 months of discharge. Significant decreases in plasma IL-4, IL-8 and eotaxin levels were also observed in the clarithromycin group. However, a subsequent, larger, randomised, placebo-controlled trial of infants $(n=71)$ aged $<24$ months admitted to hospital for clinically-suspected viral bronchiolitis found that azithromycin $\left(10 \mathrm{mg} \cdot \mathrm{kg}^{-1}\right.$ per day) was not superior to placebo with regard to length of hospital stay (the primary outcome), days of symptoms, duration of fever, bronchodilator use and need for supplemental oxygen [108]. As such, a recent Cochrane review found minimal evidence in support of the use of macrolides in infants with bronchiolitis [109]. Finally, SAWABUCHI et al. [110] have recently shown that the addition of clarithromycin to oseltamivir augmented secretory (s)IgA production and restored local mucosal sIgA levels in children with acute influenza, suggesting a boosting effect on the nasopharyngeal mucosal immune response in children with influenza A.

\section{Risks of long-term macrolide treatment}

Three major categories of adverse effects may complicate the long-term use of macrolides: ototoxicity, cardiac toxicity and drug-drug interactions. AlBERT et al. [80] reported an excess rate of hearing decrements of $\sim 5 \%$ attributable to azithromycin use. Ototoxicity following long-term azithromycin therapy has also been reported in patients with disseminated Mycobacterium avium disease [111]. Of note, replacement of azithromycin with clarithromycin in the treatment regimen led to complete recovery in patients previously complaining of hearing loss. Macrolides prolong the QTc interval [112], which, in turn, increases the risk of torsades de pointes, potentially resulting in ventricular fibrillation and sudden death. This risk is particularly high in older COPD patients who are more likely both to have cardiac disease and to be taking other drugs that prolong the QTc interval. Although erythromycin and clarithromycin have been most commonly associated with cardiac arrhythmias [113], azithromycin should also be avoided in subjects with a high risk of baseline cardiovascular disease [114]. Macrolides also inhibit the cytochrome P450 (CYP)3A4 isoenzyme, resulting in increased serum levels of other drugs metabolised by this enzyme, such as statins, warfarin and amiodarone.

Reduced susceptibility of respiratory pathogens to macrolides has increased considerably over the last decade [115]. A similar increase has also been observed in the oro-pharyngeal carriage of macrolideresistant commensals, which, although usually harmless, can cause infection in immunocompromised hosts, or transfer the resistance acquired to other pathogens [116]. Data from studies in patients with CF suggest that macrolides resistance of respiratory pathogens, particularly $S$. aureus, increases significantly when maintenance therapy is given for a long period of time (3-5 years), although without apparent adverse consequences for the treatment of subsequent acute exacerbations [117-119]. A related concern is the potential wider spread of macrolide-resistant organisms from patients being treated prophylactically both to the general population and to patients with diseases such as nontuberculous mycobacteria diseases, for which macrolides are commonly used. In this regard, ongoing research is exploring the possibility to develop macrolides that lack antimicrobial properties but retain their immunomodulatory properties, thus decreasing the risk of the development of antimicrobial resistance.

A number of recent studies have also identified an increase in mycobacterial infection of CF patients, predominantly with the multidrug-resistant highly pathogenic nontuberculous Mycobacterium abscessus [120, 121]. RENNA et al. [122] demonstrated that azithromycin paradoxically inhibits intracellular killing of mycobacteria by blocking autophagy, a critical cell homeostatic process that protects against infectious, autoimmune and inflammatory diseases.

\section{Concluding remarks}

The introduction of chronic low-dose macrolide therapy for the treatment of DPB has dramatically altered the natural history of this disease. Based on this observation, the interest in the potential use of macrolides for the treatment of chronic respiratory disease has greatly increased, and a number of studies are currently 
underway [123]. In fact, owing to their immunomodulatory and antimicrobial properties together with the high concentrations achieved in the respiratory tract tissues and extracellular fluids, macrolides represent ideal candidates for the management of lung diseases with a chronic inflammatory and infectious component [124]. The best evidence for the chronic use of macrolides is in CF. For some of these disorders, such as asthma and non-CF bronchiectasis, scientific evidence to justify the routine use of macrolides is controversial. Conversely, recent data from large clinical trials in COPD, a disease characterised by increased pulmonary inflammation at baseline, frequent bacterial colonisation/infection and recurrent exacerbations which further increase lung inflammation, suggest a beneficial effect of macrolide prophylaxis in patients at high risk of exacerbations. Nevertheless, which patients are likely to respond to macrolide therapy, the appropriate dose (once daily, three times weekly or once weekly) and duration of treatment are unknown. As such, the decision to use macrolides, with the exception of DPB (and probably also CF) should be made on a case-by-case basis. The extent of any benefit must be set against the risks of increased bacterial resistance, and whether benefits are maintained over the long term needs to be elucidated. Additional studies are needed before macrolides become an established part of routine therapy in clinical practice in cases other than in DPB and CF.

\section{References}

1 Mazzei T, Mini E, Novelli A, et al. Chemistry and mode of action of macrolides. J Antimicrob Chemother 1993; 31 : $1-9$.

2 Bearden DT, Rodvold KA. Penetration of macrolides into pulmonary sites of infection. Infect Med 1999; 16: 480-484.

3 Seppälä H, Klaukka T, Vuopio-Varkila J, et al. The effect of changes in the consumption of macrolide antibiotics on erythromycin resistance in Group A streptococci in Finland. N Engl J Med 1997; 337: 441-446.

4 Kudoh S, Azuma A, Yamamoto M, et al. Improvement of survival in patients with diffuse panbronchiolitis treated with low-dose erythromycin. Am J Respir Crit Care Med 1998; 157: 1829-1832.

5 Rubin BK. Immunomodulatory properties of macrolides: overview and historical perspective. Am J Med 2004; 117: Suppl. 9A, 2-4.

6 Tamaoki J, Takeyama K, Tagaya E, et al. Effect of clarithromycin on sputum production and its rheological properties in chronic respiratory tract infections. Antimicrob Agents Chemother 1995; 39: 1688-1690.

7 Takizawa H, Desaki M, Ohtoshi T, et al. Erythromycin modulates IL-8 expression in normal and inflamed human bronchial epithelial cells. Am J Respir Crit Care Med 1997; 156: 266-271.

8 Desaki M, Okazaki H, Sunazuka T, et al. Molecular mechanisms of anti-inflammatory action of erythromycin in human bronchial epithelial cells: possible role in the signaling pathway that regulates nuclear factor- $\mathrm{\kappa B}$ activation. Antimicrob Agents Chemother 2004; 48: 1581-1585.

9 Kusano S, Kadota J, Kohno S, et al. Effect of roxithromycin on peripheral neutrophil adhesion molecules in patients with chronic lower respiratory tract disease. Respiration 1995; 62: 217-222.

10 Crosbie PA, Woodhead MA. Long-term macrolide therapy in chronic inflammatory airway disease. Eur Respir J 2009; 33: 171-181.

11 Azuma A, Kudoh S. Diffuse panbronchiolitis in East Asia. Respirology 2006; 11: 249-261.

12 Poletti V, Casoni G, Chilosi M, et al. Diffuse panbronchiolitis. Eur Respir J 2006; 28: 862-871.

13 Ichikawa Y, Koga H, Tanaka M, et al. Neutrophilia in bronchoalveolar lavage fluid of diffuse panbronchiolitis. Chest 1990; 98: 917-923.

14 Sakito O, Kadota J, Kohno S. Interleukin $1 \beta$, tumour necrosis factor $\alpha$, and interleukin 8 in bronchoalveolar lavage fluid of patients with diffuse panbronchiolitis: a potential mechanism of macrolide therapy. Respiration 1996; 63: $42-48$.

15 Fujii T, Kadota J, Kawakami K, et al. Long term effect of erythromycin therapy in patients with chronic Pseudomonas aeruginosa infection. Thorax 1995; 50: 1246-1252.

16 Nagai H, Shishido H, Yoneda R, et al. Long-term low-dose administration of erythromycin to patients with diffuse panbronchiolitis. Respiration 1991; 58: 145-149.

17 Nakamura H, Fujishima S, Inoue T, et al. Clinical and immunoregulatory effects of roxithromycin therapy for chronic respiratory tract infection. Eur Respir J 1999; 13: 1371-1379.

18 Kadota J, Mukae H, Ishii H, et al. Long-term efficacy and safety of clarithromycin treatment in patients with diffuse panbronchiolitis. Respir Med 2003; 97: 844-850.

19 Li H, Zhou Y, Fan F, et al. Effect of azithromycin on patients with diffuse panbronchiolitis: retrospective study of 521 cases. Intern Med 2011; 50: 1663-1669.

20 Koyama H, Geddes DM. Erythromycin and diffuse panbronchiolitis. Thorax 1997; 52: 915-918.

21 Akira M, Higashihara T, Sakatani M, et al. Diffuse panbronchiolitis: follow-up CT examination. Radiology 1993; 189: $559-562$.

22 Yang M, Dong BR, Lu J, et al. Macrolides for diffuse panbronchiolitis. Cochrane Database Syst Rev 2010; 12: CD007716.

23 Davies JC, Alton EW, Bush A. Cystic fibrosis. BMJ 2007; 335: 1255-1259.

24 Gibson RL, Burns JL, Ramsey BW. Pathophysiology and management of pulmonary infections in cystic fibrosis. Am J Respir Crit Care Med 2003; 168: 918-951.

25 Cohen-Cymberknoh M, Shoseyov D, Kerem E. Managing cystic fibrosis: strategies that increase life expectancy and improve quality of life. Am J Respir Crit Care Med 2011; 183: 1463-1471.

26 Razvi S, Quittell L, Sewall A, et al. Respiratory microbiology of patients with cystic fibrosis in the United States, 1995 to 2005. Chest 2009; 136: 1554-1560.

27 Waters V, Yau Y, Prasad S, et al. Stenotrophomonas maltophilia in cystic fibrosis: serologic response and effect on lung disease. Am J Respir Crit Care Med 2011; 183: 635-640. 
28 Zlosnik JEA, Costa PS, Brant R, et al. Mucoid and nonmucoid Burkolderia cepacia complex bacteria in cystic fibrosis infections. Am J Respir Crit Care Med 2011; 183: 67-72.

29 Foweraker J. Recent advances in the microbiology of respiratory tract infection in cystic fibrosis. Br Med Bull 2009; 89: 93-110.

30 Khan TZ, Wagener JS, Bost T, et al. Early pulmonary inflammation in infants with cystic fibrosis. Am J Respir Crit Care Med 1995; 151: 1075-1082.

31 Wolter J, Seeney S, Bell S, et al. Effect of long term treatment with azithromycin on disease parameters in cystic fibrosis: a randomized trial. Thorax 2002; 57: 212-216.

32 Equi A, Balfour-Lynn IM, Bush A, et al. Long term azithromycin in children with cystic fibrosis: a randomized, placebo-controlled crossover trial. Lancet 2002; 360: 978-984.

33 Saiman L, Marshall BC, Mayer-Hamblett N, et al. Azithromycin in patients with cystic fibrosis chronically infected with Pseudomonas aeruginosa: a randomized controlled trial. JAMA 2003; 290: 1749-1756.

34 Clement A, Tamalet A, Leroux E, et al. Long term effects of azithromycin in patients with cystic fibrosis: a double blind, placebo controlled trial. Thorax 2006; 61: 895-902.

35 McCormack J, Bell S, Senini S, et al. Daily versus weekly azithromycin in cystic fibrosis patients. Eur Respir J 2007; 30: 487-495.

36 Saiman L, Anstead M, Mayer-Hamblett N, et al. Effect of azithromycin on pulmonary function in patients with cystic fibrosis uninfected with Pseudomonas aeruginosa: a randomized controlled trial. JAMA 2010; 303: 1707-1715.

37 Saiman L, Mayer-Hamblett N, Anstead M, et al. Open-label, follow-on study of azithromycin in paediatric patients with CF uninfected with Pseudomonas aeruginosa. Pediatr Pulmonol 2012; 47: 641-648.

38 Ratjen F, Saiman L, Mayer-Hamblett N, et al. Effect of azithromycin on systemic markers of inflammation in cystic fibrosis patients uninfected with Pseudomonas aeruginosa. Chest 2012; 142: 1259-1266.

39 Martinez FJ, Curtis JL, Albert R. Role of macrolide therapy in chronic obstructive pulmonary disease. Int J Chron Obstruct Pulmon Dis 2008; 3: 331-350.

40 Southern KW, Barker PM, Solis-Moya A, et al. Macrolide antibiotics for cystic fibrosis. Cochrane Database Syst Rev 2011; 11: CD002203.

41 Robinson P, Schechter MS, Sly PD, et al. Clarithromycin therapy for patients with cystic fibrosis: a randomized controlled trial. Pediatr Pulmonol 2012; 47: 551-557.

42 Barker AF. Bronchiectasis. N Engl J Med 2002; 346: 1383-1393.

43 Gaga $\mathrm{M}$, Bentley $\mathrm{AM}$, Humbert $\mathrm{M}$, et al. Increases in $\mathrm{CD}^{+} \mathrm{T}$ lymphocytes, macrophages, neutrophils and interleukin 8 positive cells in the airways of patients with bronchiectasis. Thorax 1998; 53: 685-691.

44 Koh YY, Lee MH, Sun YH, et al. Effect of roxithromycin on airway responsiveness in children with bronchiectasis: a double-blind, placebo-controlled study. Eur Respir J 1997; 10: 994-999.

45 Tsang KW, Ho PI, Chan KN, et al. A pilot study of low-dose erythromycin in bronchiectasis. Eur Respir J 1999; 13: 361-364.

46 Yalçin E, Kiper N, Ozçelik U, et al. Effects of clarithromycin on inflammatory parameters and clinical conditions in children with bronchiectasis. J Clin Pharm Ther 2006; 31: 49-55.

47 Davies G, Wilson R. Prophylactic antibiotic treatment of bronchiectasis with azithromycin. Thorax 2004; 59: 540-541.

48 Wong C, Jayaram L, Karalus N, et al. Azithromycin for prevention of exacerbations in non-cystic fibrosis bronchiectasis (EMBRACE): a randomized, double-blind, placebo-controlled trial. Lancet 2012; 380: 660-667.

49 Bateman ED, Hurd SS, Barnes PJ, et al. Global strategy for asthma management and prevention: GINA executive summary. Eur Respir J 2008; 31: 43-78.

50 Huang YJ, Nelson CE, Brodie EL, et al. Airway microbiota and bronchial hyperresponsiveness in patients with suboptimally controlled asthma. J Allergy Clin Immunol 2011; 127: 372-381.

51 Specjalski K, Jassem E. Chlamydophila pneumoniae, Mycoplasma pneumoniae infections, and asthma control. Allergy Asthma Proc 2011; 32: 9-17.

52 Kostadima E, Tsiodras S, Alexopoulos EI, et al. Clarithromycin reduces the severity of bronchial hyperresponsiveness in patients with asthma. Eur Respir J 2004; 23: 714-717.

53 Zeiger RS, Schatz M, Sperling W, et al. Efficacy of troleandomycin in outpatients with severe, corticosteroiddependent asthma. J Allergy Clin Immunol 1980; 66: 438-446.

54 Kamada AK, Hill MR, Ikle DN, et al. Efficacy and safety of low-dose troleandomycin therapy in children with severe, steroid-requiring asthma. J Allergy Clin Immunol 1993; 91: 873-882.

55 Nelson HS, Hamilos DL, Corsello PR, et al. A double-blind study of troleandomycin and methylprednisolone in asthmatic subjects who require daily corticosteroids. Am Rev Respir Dis 1993; 147: 398-404.

56 Black PN, Blasi F, Jenkins CR, et al. Trial of roxithromycin in subjects with asthma and serological evidence of infection with Chlamydia pneumoniae. Am J Respir Crit Care Med 2001; 164: 536-541.

57 Kraft M, Cassell GH, Pak J, et al. Mycoplasma pneumoniae and Chlamydia pneumoniae in asthma: effect of clarithromycin. Chest 2002; 121: 1782-1788.

58 Sutherland ER, King TS, Icitovic N, et al. A trial of clarithromycin for the treatment of suboptimally controlled asthma. J Allergy Clin Immunol 2010; 126: 747-753.

59 Richeldi L, Ferrara G, Fabbri LM, et al. Macrolides for chronic asthma. Cochrane Database Syst Rev 2005; 4: CD002997.

60 Strunk RC, Bacharier LB, Phillips BR, et al. Azithromycin or montelukast as inhaled corticosteroid-sparing agents in moderate-to-severe childhood asthma study. J Allergy Clin Immunol 2008; 122: 1138-1144.

61 Mathers CD, Boerma T, Ma Fat D. Global and regional causes of death. Br Med Bull 2009; 92: 7-32.

62 Vestbo J, Edwards LD, Scanlon PD, et al. Changes in forced expiratory volume in 1 second over time in COPD. N Engl J Med 2011; 65: 1184-1192.

63 Soler-Cataluna JJ, Martinez-Garcia MA, Roman Sanchez P, et al. Severe acute exacerbations and mortality in patients with chronic obstructive pulmonary disease. Thorax 2005; 60: 925-931.

64 Seemungal T, Donaldson GC, Paul EA, et al. Effect of exacerbation on quality of life in patients with chronic obstructive pulmonary disease. Am J Respir Crit Care Med 1998; 157: 1418-1422.

65 Miravitlles M, Murio C, Guerrero T, et al. Pharmacoeconomic evaluation of acute exacerbations of chronic bronchitis and COPD. Chest 2002; 121: 1449-1455. 
66 Burge PS, Calverley PMA, Jones PW, et al. Randomised, double blind, placebo controlled study of fluticasone propionate in patients with moderate to severe chronic obstructive pulmonary disease: the ISOLDE trial. $B M J 2000$; 320: 1297-1303.

67 Calverley PMA, Anderson JA, Celli B, et al. Salmeterol and fluticasone propionate and survival in chronic obstructive pulmonary disease. N Engl J Med 2007; 356: 775-789.

68 Tashkin DP, Celli B, Senn S, et al. A 4-year trial of tiotropium in chronic obstructive pulmonary disease. $N$ Engl J Med 2008; 359: 1543-1554.

69 Welte T, Miravitlles M, Hernandez P, et al. Efficacy and tolerability of budesonide/formoterol added to tiotropium in patients with chronic obstructive pulmonary disease. Am J Respir Crit Care Med 2009; 180: 741-750.

70 Bateman ED, Rabe KF, Calverley PM, et al. Roflumilast with long-acting $\beta_{2}$-agonists for COPD: influence of exacerbation history. Eur Respir J 2011; 38: 553-560.

71 Aaron SD, Vandemheen KL, Fergusson D, et al. Tiotropium in combination with placebo, salmeterol, or fluticasone-salmeterol for treatment of chronic obstructive pulmonary disease: a randomized trial. Ann Intern Med 2007; 146: 545-555.

72 Yamaya M, Azuma A, Takizawa H, et al. Macrolide effects on the prevention of COPD exacerbations. Eur Respir J 2012; 40: 485-494

73 Yamaya M, Azuma A, Tanaka $\mathrm{H}$, et al. Inhibitory effects of macrolide antibiotics on exacerbations and hospitalization in chronic obstructive pulmonary disease in Japan: a retrospective multicenter analysis. J Am Geriatr Soc 2008; 56: 1358-1360.

74 Banerjee D, Khair OA, Honeybourne D. The effect of oral clarithromycin on health status and sputum bacteriology in stable COPD. Respir Med 2005; 99: 208-215.

75 Suzuki T, Yanai M, Yamaya M, et al. Erythromycin and common cold in COPD. Chest 2001; 120: 730-733.

76 Blasi F, Bonardi D, Aliberti S, et al. Long-term azithromycin use in patients with chronic obstructive pulmonary disease and tracheostomy. Pulm Pharmacol Ther 2010; 3: 200-207.

77 Gómez J, Baños V, Simarro E, et al. Estudio prospective y comparativo (1994-1998) sobre la influencia corto profiláctico con azitromicina en pacientes con EPOC evolucionada. [Prospective, comparative study (1994-1998) of the influence of short-term prophylactic treatment with azithromycin on patients with advanced COPD.]. Rev Esp Quimioter 2000; 13: 379-383.

78 He ZY, Ou LM, Zhang JQ, et al. Effect of 6 months of erythromycin treatment on inflammatory cells in induced sputum and exacerbations in chronic obstructive pulmonary disease. Respiration 2010; 80: 445-452.

79 Seemungal TA, Wilkinson TM, Hurst JR, et al. Long-term erythromycin therapy is associated with decreased chronic obstructive pulmonary disease exacerbations. Am J Respir Crit Care Med 2008; 178: 1139-1147.

80 Albert RK, Connett J, Bailey WC, et al. Azithromycin for prevention of exacerbations of COPD. N Engl J Med 2011; 365: 689-698.

81 Sethi S, Murphy TF. Infection in the pathogenesis and course of chronic obstructive pulmonary disease. $N$ Engl J Med 2008; 359: 2355-2365.

82 Global Strategy for the Diagnosis, Management and Prevention of COPD, Global Initiative for Chronic Obstructive Lung Disease (GOLD) 2013. Available from: www.goldcopd.org.

83 Marras T, Chan C. Obliterative bronchiolitis complicating bone marrow transplantation. Semin Respir Crit Care Med 2003; 24: 531-541.

84 Gottlieb J, Szangolies J, Koehnlein T, et al. Long-term azithromycin for bronchiolitis obliterans syndrome after lung transplantation. Transplantation 2008; 85: 36-41.

85 Verleden GM, Vanaudenaerde BM, Dupont LJ, et al. Azithromycin reduces airway neutrophilia and interleukin-8 in patients with bronchiolitis obliterans syndrome. Am J Respir Crit Care Med 2006; 174: 566-570.

86 Vanaudenaerde BM, Meyts I, Vos R, et al. A dichotomy in bronchiolitis obliterans syndrome after lung transplantation revealed by azithromycin therapy. Eur Respir J 2008; 32: 832-843.

87 Jain R, Hachem RR, Morrell MR, et al. Azithromycin is associated with increased survival in lung transplant recipients with bronchiolitis obliterans syndrome. J Heart Lung Transplant 2010; 29: 531-537.

88 Collins JG. Prevalence of selected chronic conditions: United States, 1990-1992. Vital Health Stat 1997; 194: 1-89.

89 Hashiba M, Baba S. Efficacy of long-term administration of clarithromycin in the treatment of intractable chronic sinusitis. Acta Otolaryngol Suppl 1996; 525: 73-78.

90 Kimura N, Nishioka K, Nishizaki K, et al. Clinical effect of low-dose, long-term roxithromycin chemotherapy in patients with chronic sinusitis. Acta Med Okayama 1997; 51: 33-37.

91 Suzuki H, Shimomura A, Ikeda K. Effects of long-term low-dose macrolide administration on neutrophil recruitment and IL-8 in the nasal discharge of chronic sinusitis patients. Tohoku J Exp Med 1997; 182: 115-124.

92 Yamada T, Fujieda S, Mori S, et al. Macrolide treatment decreased the size of nasal polyps and IL-8 levels in nasal lavage. Am J Rhinol 2000; 14: 143-148.

93 Suzuki H, Takahashi Y, Wataya H, et al. Mechanism of neutrophil recruitment induced by IL-8 in chronic sinusitis. J Allergy Clin Immunol 1996; 98: 659-970.

94 Tamaoki J. The effects of macrolides on inflammatory cells. Chest 2004; 125: 41S-50S.

95 Videler WJ, Badia L, Harvey RJ, et al. Lack of efficacy of long-term, low-dose azithromycin in chronic rhinosinusitis: a randomized controlled trial. Allergy 2011; 66: 1457-1468.

96 Fokkens WJ, Lund VJ, Mullol J, et al. European position paper on rhinosinusitis and nasal polyps 2012. Rhinol Suppl 2012; 23: 1-298.

97 Wuyts WA, Willems S, Vos R, et al. Azithromycin reduces pulmonary fibrosis in a bleomycin mouse model. Exp Lung Res 2010; 36: 602-614.

98 Guillot L, Tabary O, Nathan N, et al. Macrolides: new therapeutic perspectives in lung diseases. Int J Biochem Cell Biol 2010; 43: 1241-1246.

99 Cordier JF. Cryptogenic organising pneumonia. Eur Respir J 2006; 28: 422-446.

100 Stover DE, Mangino D. Macrolides: a treatment alternative for bronchiolitis obliterans organizing pneumonia? Chest 2005; 128: 3611-3617.

101 Hotta M. Neutrophil chemotactic activity in cryptogenic organizing pneumonia and the response to erythromycin. Kurume Med J 1996; 43: 207-217. 
102 Rubenfeld GD, Caldwell E, Peabody E, et al. Incidence and outcomes of acute lung injury. N Engl J Med 2005; 353: $1685-1693$.

103 Walkey AJ, Wiener RS. Macrolide antibiotics and survival in patients with acute lung injury. Chest 2012; 141: 1153-1159.

104 Hall CB, Weinberg GA, Iwane MK, et al. The burden of respiratory syncytial virus infection in young children. N Engl J Med 2009; 360: 588-598.

105 Subcommittee on Diagnosis and Management of Bronchiolitis. Diagnosis and management of bronchiolitis. Pediatrics 2006; 118: 1774-1793.

106 Lee SM, Gardy JL, Cheung CY, et al. Systems-level comparison of host-responses elicited by avian H5N1 and seasonal H1N1 influenza viruses in primary human macrophages. PLoS One 2009; 4: e8072.

107 Tahan F, Ozcan A, Koc N. Clarithromycin in the treatment of RSV bronchiolitis: a double-blind, randomised, placebo-controlled trial. Eur Respir J 2007; 29: 91-97.

108 Kneyber MCJ, Van Woensel JBM, Uijtendaal E, et al. Azithromycin does not improve disease course in hospitalized infants with respiratory syncytial virus (RSV) lower respiratory tract disease: a randomized equivalence trial. Pediatr Pulmonol 2008; 43: 142-149.

109 Spurling GKP, Doust J, Del Mar CB, et al. Antibiotics for bronchiolitis in children. Cochrane Database Syst Rev 2011; 6: CD005189.

110 Sawabuchi T, Suzuki S, Iwase K, et al. Boost of mucosal secretory immunoglobulin A response by clarithromycin in paediatric influenza. Respirology 2009; 14: 1173-1179.

111 Wallace MR, Miller LK, Nguyen MT, et al. Ototoxicity with azithromycin. Lancet 1994; 343: 241.

112 Volberg WA, Koci BJ, Su W, et al. Blockade of human cardiac potassium channel human ether-a-go-go-related gene (HERG) by macrolide antibiotics. J Pharmacol Exp Ther 2002; 302: 320-327.

113 Simko J, Csilek A, Karaszi J, et al. Proarrhythmic potential of antimicrobial agents. Infection 2008; 36: 194-206.

114 Ray WA, Murray KT, Hall K, et al. Azithromycin and the risk of cardiovascular death. N Engl J Med 2012; 366: $1881-1890$.

115 Bergman M, Huikko S, Huovinen P, et al. Macrolide and azithromycin use are linked to increased macrolide resistance in Streptococcus pneumoniae. Antimicrob Agents Chemother 2006; 50: 3646-3650.

116 Lonks JR, Garau J, Medeiros AA. Implications of antimicrobial resistance in the empirical treatment of communityacquired respiratory tract infections: the case of macrolides. J Antimicrob Chemother 2002; 50: 87-92.

117 Phaff SJ, Tiddens HA, Verbrugh HA, et al. Macrolide resistance of Staphylococcus aureus and Haemophilus species associated with long-term azithromycin use in cystic fibrosis. J Antimicrob Chemother 2006; 57: 741-746.

118 Tramper-Stranders GA, Wolfs TF, Fleer A, et al. Maintenance azithromycin treatment in pediatric patients with cystic fibrosis: long-term outcomes related to macrolide resistance and pulmonary function. Pediatr Infect Dis $J$ 2007; 26: 8-12.

119 K.asahara K, Kita E, Maeda K, et al. Macrolide resistance of Streptococcus pneumoniae isolated during long-term macrolide therapy: difference between erythromycin and clarithromycin. J Infect Chemother 2005; 11: 112-114.

120 Esther CR Jr, Esserman DA, Gilligan P, et al. Chronic Mycobacterium abscessus infection and lung function decline in cystic fibrosis. J Cyst Fibros 2010; 9: 117-123.

121 Roux AL, Catherinot E, Ripoll F, et al. Multicenter study of prevalence of nontuberculous mycobacteria in patients with cystic fibrosis in France. J Clin Microbiol 2009; 47: 4124-4128.

122 Renna M, Schaffner C, Brown K, et al. Azithromycin blocks autophagy and may predispose cystic fibrosis patients to mycobacterial infection. J Clin Invest 2011; 121: 3554-3563.

123 US National Institutes of Health. ClinicalTrials.gov. http://clinicaltrials.gov/ct2/results?term=respiratory+diseases +AND+macrolides\&pg =2 Date last accessed: May 7, 2013. Date last updated: May 3, 2013.

124 Jain R, Danziger LH. The macrolide antibiotics: a pharmacokinetic and pharmacodynamic overview. Curr Pharm Des 2004; 10: 3045-3053. 